\title{
STUDY OF PREVALENCE OF TUBERCULOSIS AND RIFAMPICIN RESISTANT TUBERCULOSIS AMONG THE SAMPLE RECEIVED AT CB-NAAT CENTER IN A TERTIARY CARE HOSPITAL ANMMCH, GAYA
}

\author{
Ramesh Prasad Singh', Ankur Kumar², Arjun Lal ${ }^{3}$, Sushil Prasad Mahto ${ }^{4}$
}

1Tutor, Department of Microbiology, Anugrah Narayan Magadh Medical College and Hospital, Gaya, Bihar, India.

2Tutor, Department of Microbiology, Anugrah Narayan Magadh Medical College and Hospital, Gaya, Bihar, India.

${ }^{3}$ Associate Professor, Department of Microbiology, Anugrah Narayan Magadh Medical College and Hospital, Gaya, Bihar, India.

4 Professor, Department of Microbiology, Anugrah Narayan Magadh Medical College and Hospital, Gaya, Bihar, India.

\begin{abstract}
\section{BACKGROUND}

Tuberculosis (TB) remains a leading health problem not only in India but all over the world. India is the country with highest TB burden accounting for one-fourth (25\%) of global TB cases. Diagnosis of tuberculosis (Pulmonary and Extra Pulmonary TB) still remains a challenge. By using the traditional method for diagnosing TB like tuberculin test, chest radiography and/or sputum microscopy, the result was not so convincing. In sputum microscopy, the yield of identifying TB bacilli is very low especially in paediatric TB due to the paucibacillary nature. Thus, diagnosis of TB especially in immunocompromised patients, sputum-smear negative or paediatric cases is more problematic and challenging. By WHO in December 2010, Xpert $®$ MTB/RIF assay (Cepheid, Sunnyvale CA, USA) was recommended, based on real-time nucleic acid amplification technology and automated. Xpert@ MTB/RIF assay done with barcoded cartridge, so this technique is also called cartridge based nucleic acid amplification test (CB-NAAT). This study assessed the applicability of (Gene Xpert) in early diagnosis of TB, and early identification of MDR-TB. GeneXpert is one of the recent technological instruments used to diagnose tuberculosis in a short span of time.
\end{abstract}

\section{METHODS}

This was a cross sectional study, carried out on all patients of who were suspected cases of tuberculosis, visiting the OPD or indoor at tertiary care hospital ANMMCH, Gaya, over a period of 3 months from 01-10-2017 to 31-12-2017. A total of 489 patients were sent for CB-NAAT center.

\section{RESULTS}

Out of 489 suspected patients, 127 (25.97\%) were detected positive for MTB. Among the 127 MTB detected cases 102 (80.31\%) were Rifampicin sensitive and Rifampicin resistance was 25 (19.68\%).

\section{CONCLUSIONS}

This study makes us aware of the high burden of tuberculosis cases and higher risk of MDR-TB in this local region. It also helps in early institution of first line anti-tubercular therapy and screening of MDR-TB for further second line anti-TB drugs therapy to get an earlier and better outcome.

\section{KEY WORDS}

Tuberculosis, CB-NAAT, Rifampicin Resistant Tuberculosis, MDR- TB

HOW TO CITE THIS ARTICLE: Singh RP, Kumar A, Lal A, et al. Study of prevalence of tuberculosis and rifampicin resistant tuberculosis among the sample received at CB-NAAT center in a tertiary care hospital ANMMCH, Gaya. J. Evolution Med. Dent. Sci. 2019;8(24):1891-1895, DOI: 10.14260/jemds/2019/416

\section{BACKGROUND}

Tuberculosis (TB) is the most ancient of diseases of human being. The molecular evidence of TB was detected first time in a fossil of an extinct bison (Pleistocene bison)- detected by radiocarbon dated at $17,870 \pm 230$ years ago,(2) and in the Eastern Mediterranean 9000 years old human remains were recovered from a neolithic settlement. (3)

But TB was first established by Dr. Richard Morton in 1689 , it was the pulmonary form of "tubercles," because of all the symptoms are associated with Lung infection. TB was still

'Financial or Other Competing Interest': None.

Submission 04-04-2019, Peer Review 04-06-2019,

Acceptance 10-06-2019, Published 17-06-2019.

Corresponding Author:

Dr. Ankur Kumar,

C/o. Ashok Kr. Singh,

Chankya Nagar, Kumhrar,

Near Lalita Raj Appt.,

Patna-800026, Bihar, India.

E-mail: ankur29nov@gmail.com

DOI: $10.14260 /$ jemds $/ 2019 / 416$ not identified as a single disease until the 1820s. Thereafter in 1839 by J. L. Schönlein all these presentations of lung infection were eventually named as "tuberculosis.(4) In 1882, Robert Koch was discovered the bacillus which causing tuberculosis Mycobacterium tuberculosis; and he was awarded Nobel prize in physiology or medicine in 1905 for this discovery.(5)

In Indian scenario, TB is mentioned in the various old scriptures like - Vedas and the Ayurvedic. Mycobacterium tuberculosis complex is a closely related mycobacterial species which is associated with Tuberculosis infections. It usually affects the lungs, although other organs are also involved. Mycobacterium tuberculosis complex includes: M. tuberculosis - called human tubercle bacillus, M. bovis bovine tubercle bacillus, $M$. caparae closely related to $M$. bovis, M. africanum -isolated from few West African cases, M. microti - is 'vole' bacillus, rare and less virulent, M. pinnipedii - infects seals in die Southern hemisphere and recently isolated from humans, $M$. canetti - a rare isolate from East African cases, that produces unusual smooth colonies on solid media. Among all, M. tuberculosis is the most common 
cause of tuberculosis in man. M. bovis, M. microti and M. africanum are the other members of the M. tuberculosis complex that can cause tuberculosis, but these are not associated with TB in human beings.

\section{Primary Infection and Reactivation}

When a host has first contact with tubercle bacilli, various features are observed-

1- An acute exudative lesion develops and rapidly spreads to the lymphatics (Lymphangitis) and regional lymph nodes (Lymphadenitis). The exudative lesion in tissue often heals rapidly.

2- The lymph node undergoes massive caseation, which usually calcifies (Ghon lesion).

3- The tuberculin test result becomes positive. This primary infection type occurred in the past, usually in childhood, but now frequently in adults who have remained free from infection and therefore tuberculin negative in early life. In primary infections, the involvement may be in any part of the lung but is most often at the base.

The reactivation type is usually caused by tubercle bacilli that have survived in the primary lesion. Reactivation tuberculosis is characterized by chronic tissue lesions, the formation of tubercles, caseation, and fibrosis. Regional lymph nodes are only slightly involved, and they do not caseate. The reactivation type almost always begins at the apex of the lung, where the oxygen tension $\left(\mathrm{PO}_{2}\right)$ is highest. These differences between primary infection and reinfection or reactivation are attributed to 1- resistance and 2hypersensitivity induced by the first infection. It is not clear to what extent each of these components participates in the modified response in reactivation tuberculosis.

\section{Clinical Findings}

Because the tubercle bacillus can involve every organ system, its clinical manifestations represent slight variations depends upon the site of involvement. But non-specific symptoms of tuberculous disease are - fatigue, weakness, weight loss, fever, and night sweats. Pulmonary involvement giving rise to chronic cough and spitting of blood usually is associated with far-advanced lesions. Meningitis or urinary tract involvement can occur in the absence of other signs of tuberculosis. Bloodstream dissemination leads to miliary tuberculosis with lesions in many organs and a high mortality rate.

\section{Clinical Manifestations}

Tuberculosis (TB) is classified in to two forms - pulmonary and extrapulmonary forms. Pulmonary Tuberculosis (PTB)accounts for $80 \%$ of all cases of Tuberculosis (TB). It can be further categorized into primary or post-primary (secondary) types. Extrapulmonary Tuberculosis (EPTB)- EPTB results from hematogenous dissemination of tubercle bacilli to various organs other than lung. Though EPTB constitutes about $15-20 \%$ of all cases of TB, in HIV-positive patients, the frequency is much higher accounting for $20-50 \%$ of all cases of tuberculosis. EPTB form virtually involved all organ systems of human bodies but commonly involved sites and names are lymph nodes called Tuberculous lymphadenitis constitutes $35 \%$ of all EPTB cases, Pleural tuberculosis constitutes $20 \%$ of all EPTB cases, Tuberculosis of the upper airways- involving larynx, pharynx, and epiglottis,
Genitourinary tuberculosis- consists Renal tuberculosis and Genital tuberculosis, Skeletal tuberculosis, Tuberculosis of CNS, Gastrointestinal tuberculosis, Tuberculous pericarditis, Tuberculous skin lesions- Scrofuloderma, Lupus vulgaris, Miliary or disseminated tuberculosis- mainly associated with HIV-infected people. Globally TB occurs in 70--80\% of HIVinfected individuals and EPTB forms are being more common than PTB in HIV infected individuals.

\section{Now a Days}

Tuberculosis (TB) remains a leading health problem not only in India but also in all over the world, that is globally. About one-third of the current world population is infected asymptomatically with $M$. tuberculosis, of which 5- $10 \%$ develop clinical disease during their lifetime. WHO has estimated data in the year 2010- that 8.8 million new cases of TB occurred worldwide in 2010, $95 \%$ of them in developing countries of Asia and Africa. The number of people dying from tuberculosis in 2010 was about 1.4 million. Patients with infectious pulmonary TB can infect 10 - 15 people in a year.

The epidemics of TB is more than the incidence, which is 10.4 million new TB cases, previously estimated worldwide in 2015. Out of 10.4 million new TB cases - 5.9 million (56\%) were men, 3.5 million (34\%) were women and 1.0 million $(10 \%)$ children.(6) India have major impact over the global TB burden and its accounts for one fourth of total TB burden globally. An estimated data in the year of 2015- 2, 800, 000 TB cases occurred and among them 480, 000 people were died due to TB. According to the Global TB Report 2016 India have estimated the highest burden of both TB and MDR-TB, out of 0.48 million of MDR- TB cases globally, 0.13 million of MDR- TB from India.(7)

India is the country with highest $\mathrm{TB}$ burden accounting for one-fourth (25\%) of global TB cases. In 2011, the prevalence and incidence rates of tuberculosis in India were 256 and 185 cases per one lakh population respectively. In India, 2 deaths occur due to tuberculosis every three minutes and more than 1000 persons die every day and almost 0.37 million die every year.

With 10.4 million incident cases of tuberculosis (TB) and 1.7 million deaths estimated in 2016,(8) TB is a leading cause of morbidity and mortality worldwide. However, public health services globally reported only 6.3 million (61\%) of the estimated TB cases in 2016. Moreover, less than $5 \%$ of notified TB cases were tested for drug resistance, (8) which is often diagnosed after prolonged diagnostic delays. $(9,10,11)$ Of the 6, 00, 000 notified new and re-treatment cases with pulmonary TB estimated to have multidrug-resistant (MDR) TB in 2016, just under 1, 32, $000(22 \%)$ were reported to the World Health Organization (WHO).(8)

Main reasons for these gaps are inadequate diagnostic capacity and an over reliance on tuberculin test, chest radiography and/or sputum smear microscopy as diagnostic tools. A positive tuberculin test result does not prove the presence of active disease caused by tubercle bacilli. Isolation of tubercle bacilli provides such proof. Patients with HIVassociated $\mathrm{TB}$, those with sputum smear-negative and/or extrapulmonary disease, and drug-resistant TB patients are particularly affected by the failure of microscopy as primary diagnostic tool. The "classical" diagnosis of HIV-associated and drug-resistant TB is complex, expensive, slow and 
technically demanding, relying on conventional culture and drug susceptibility testing (DST). The long delay (Up to several weeks) required to obtain results has devastating consequences for patients who go undiagnosed (And therefore untreated or inappropriately treated) or are diagnosed too late.(12)

It is also medically important to characterize and separate Mycobacterium tuberculosis complex from all the other species of mycobacteria. Isolated mycobacteria should be identified as to species. Conventional methods for identification of mycobacteria include observation of rate of growth, colony morphology, pigmentation, and biochemical profiles. The conventional methods often require 6-8 weeks for identification and are rapidly becoming of historical interest because they are inadequate to identify the expanding numbers of clinically relevant species. Most laboratories have abandoned reliance on these biochemical tests.

Thus, for detection of more cases, detecting them early and rapidly identifying drug resistance detection are must be upgraded for improving the individual patient health and avoiding transmission in the community. This requires universal access and early detection using contemporary tools and innovative strategies. $(8,11,12)$ The last decade has seen unprecedented growth in the TB diagnostic pipeline and accelerated efforts to establish the necessary laboratory infrastructure.(12) Nevertheless, although recommended by WHO, the latest generation liquid culture diagnostics and molecular line probe assays for rapid detection of MDR-TB have not yet solved the diagnostic dilemma in most resourcelimited settings, largely due to the need for expensive laboratory infrastructure, extensive biosafety precautions, and specialized staff.(12)

According to the latest Revised National TB Control Program (RNTCP) and Indian Academy of Paediatrics (IAP) guidelines, all attempts should be made to obtain a bacteriological diagnosis.

Early detection is the key to successful treatment and reduction of the disease transmission.

\section{Newer Techniques for Detection of TB}

High-performance liquid chromatography (HPLC) has been applied to speciation of mycobacteria. The method is based on development of profiles of mycolic acids, which vary from one species to another. HPLC to speciate mycobacterium is available in reference laboratories.

Matrix-assisted laser desorption ionization-time of flight mass spectrometry (MALDI-TOF MS) has not been shown to date to be useful for accurate identification of Mycobacterium species from culture material. Susceptibility testing of mycobacteria is an important adjunct in selecting drugs for effective therapy. A standardized broth culture technique can be used to test for susceptibility to first-line drugs. The complex and more arduous conventional agar-based technique usually is performed in reference laboratories; first- and second-line drugs can be tested by this method.

Polymerase chain reaction (PCR) holds great promise for the rapid and direct detection of $\mathrm{M}$ tuberculosis in clinical specimens. The overall sensitivity is $55-90 \%$ with a specificity of about $99 \%$. The test has the highest sensitivity when applied to specimens that have smears that are positive for acid-fast bacilli.
Newer nucleic acid amplification technologies combine detection of $\mathrm{M}$ tuberculosis complex along with common resistance genes for rifampin (RMP) and other first-line drugs. These assays are not yet widely available but are available in various countries. The characterization of specific strains of $M$ tuberculosis can be important for clinical and epidemiologic purposes. It allows such things as tracing transmission from one person to another, analysis of outbreaks of tuberculosis, and demonstration of reactivation versus reinfection in individual patients. DNA fingerprinting is done using a standardized protocol based on restriction fragment length polymorphism.

A new rapid test that overcomes many of the current operational difficulties was recommended for use by WHO in December 2010 - the Xpert $\AA$ MTB/RIF assay (Cepheid, Sunnyvale CA, USA), an automated, real-time nucleic acid amplification technology run on the multi-disease platform Gene Xpert (Cepheid, Sunnyvale CA, USA). The Xpert $\AA$ $\mathrm{MTB} / \mathrm{RIF}$ assay represents a paradigm shift in the diagnosis of TB and drug-resistant TB by simultaneously detecting $\mathrm{M}$. tuberculosis and rifampicin resistance-conferring mutations, in a closed system suitable for use outside conventional laboratory settings, in less than two hours, directly from sputum samples.(11,12) The Xpert $₫$ MTB/RIF are also called Cartridge Based - Nucleic Acid Amplification Test (CB-NAAT).

\section{Aim of The Study}

To estimate the burden of tuberculosis among the suspected samples received at CB-NAAT center ANMMCH, Gaya.

\section{METHODS}

\section{Type of Study}

This study was a cross sectional study.

\section{Period of Study}

3 months period from 1-10-2017 to 31-12-2017.

\section{Place of Study}

The present study was conducted at Department of Microbiology \& the CB-NAAT center ANMMCH, Gaya.

\section{Age}

No age specification.

\section{Gender}

No gender specification.

\section{Sample Size}

During above mention 3 months' time period a total of 489 suspected specimen were received at CB-NAAT center ANMMCH, Gaya.

\section{Nature of Sample}

Fresh sputum, pleural fluid, Laryngeal swabs or bronchial washings, peritoneal fluid, pericardial fluid pus, lymph node aspirate, urine, synovial fluid, cerebrospinal fluid (CSF), endometrial/ tissue specimen or other suspected materials. Were collected from 489 different suspected tuberculosis patients visited the OPD or indoor at tertiary care hospital ANMMCH, Gaya. 

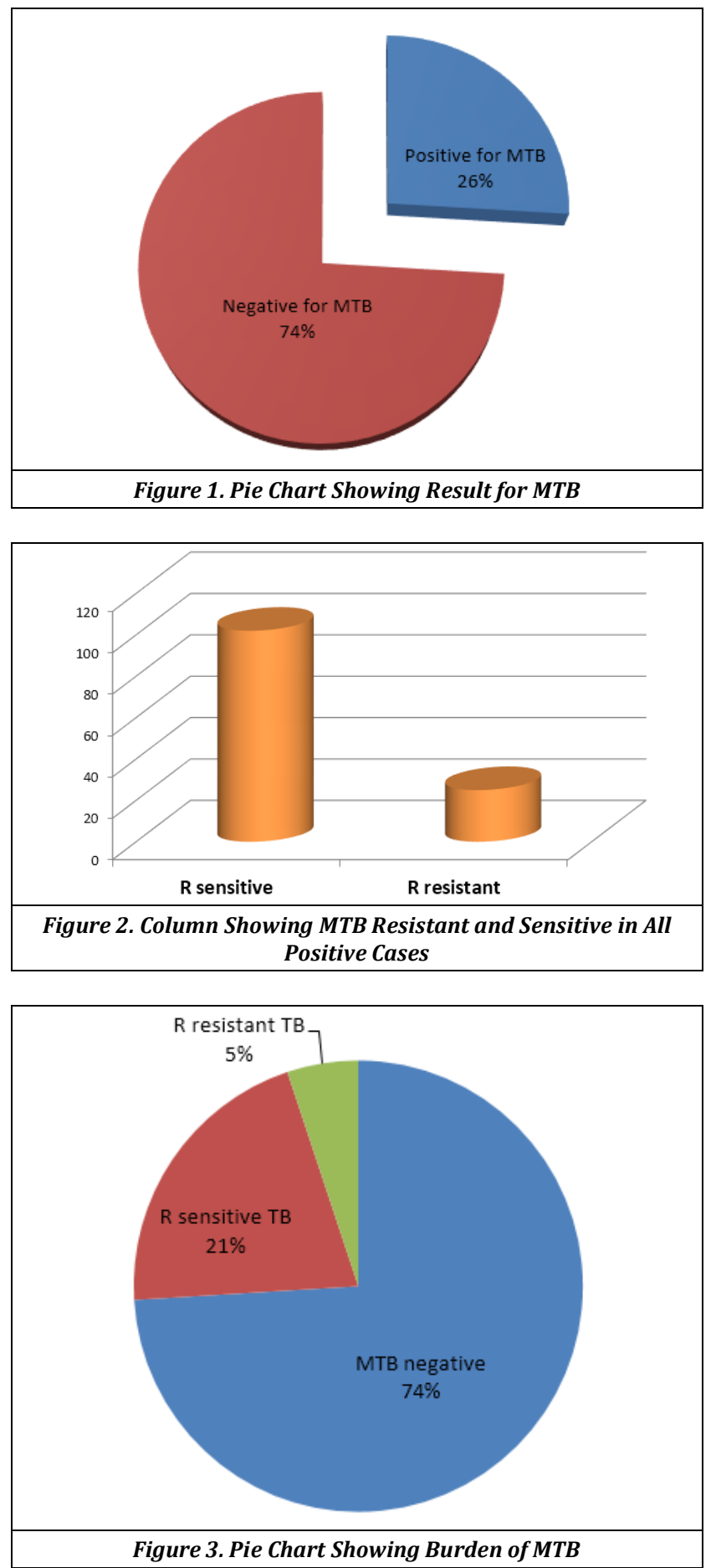

All specimens were collected in a pre-sterilized falcon tubes. ${ }^{(8)}$ These sample are received at the CB-NAAT center ANMMCH, Gaya for the diagnosis of tuberculosis and Rifampicin resistance among the positive cases of tuberculosis.

\section{Testing Procedures}

On the receipt of the specimen, the lab technician processed the specimen by adding the buffer solution in 2:1 ratio (Two times buffer against the specimen quantity) and shaking the mixture well. Stand the mixture for 10 minutes, again shake it and then stand it again for 5 more minutes. Then carefully transfer $2 \mathrm{ml}$ of the mixture to the cartridge and install the cartridge into the module as per manufacturers instructions.(13) The MTB/RIF assay interpretation is software based and not user dependent.

\section{RESULTS}

Out of 489 different specimen received at CB-NAAT center, from 489 different suspected patients visited the OPD or indoor at tertiary care hospital ANMMCH, Gaya -127(25.97\%) were detected positive for MTB (Fig. 1). Among the 127 MTB detected cases, 102 (80.31\%) were Rifampicin sensitive and 25 (19.68\%) were Rifampicin resistance (Fig. 2). Rifampicin resistance cases further repeated with another sample for confirmation and result was again resistant with Rifampicin in all repeated sample.

\section{DISCUSSION}

Actually CB-NAAT is an automated, semi-quantitative real time PCR assay designed for the rapid and simultaneous detection of Mycobacterium tuberculosis and Rifampicin resistance within 2 hours (which is much faster than any other conventional cultural method). 'Rifampicin' resistance is a surrogate marker of MDR-TB. All TB cases diagnosed with Xpert MTB/RIF and Rifampicin resistant should be registered as Xpert MTB/RIF positive with rifampicin resistance. If isoniazid resistance is confirmed by conventional or molecular techniques, the case should be registered as MDRTB.(14)

In this study burden of tuberculosis is nearly $26 \%$ among suspected patients (Fig. 1) and Rifampicin resistance is nearly $20 \%$ among positive patient (Fig. 2).

That makes aware of high burden of tuberculosis cases and higher risk of MDR-TB in this local region (Fig. 3).

There are only a few studies on CB-NAAT from India. A study done in 2011 in Hyderabad showed incremental case detection of $10.8 \%$ when CBNAAT was used to diagnose tuberculosis over and above fluorescent microscopy.(15)

\section{CONCLUSIONS}

From this study we conclude that due to advent of CB-NAAT, diagnosis of tuberculosis and Rifampicin resistant TB is earlier (Within $2 \mathrm{hrs}$.) and simpler than any other conventional cultural method. This helps in early institution of first line anti-tubercular therapy and screening of MDR TB for further second line anti-TB drugs therapy to get an earlier and better outcome.

\section{REFERENCES}

[1] Hirsh AE, Tsolaki AG, DeRiemer K, et al. Stable association between strains of Mycobacterium tuberculosis and their human host populations. Proc Natl Acad Sci USA 2004;101(14):4871-6.

[2] Rothschild BM, Martin LD, Lev G, et al. Mycobacterium tuberculosis complex DNA from an extinct bison dated 17, 000 years before the present. Clin Infec Dis 2001;33(3):305-11.

[3] Hershkovitz I, Donoghue HD, Minnikin DE, et al. Detection and molecular characterization of 9,000year-old Mycobacterium tuberculosis from a neolithic settlement in the Eastern Mediterranean. PLoS One 2008;3(10):e3426. 
[4] News-medical.net. History of Tuberculosis. [Last cited on $2010 \quad$ Oct 15]. http://www.newsmedical.net/health/History-of-Tuberculosis.aspx.

[5] Nobelprize.org. Sweden: The Nobel Prize in Physiology or Medicine 1905: Robert Koch. c2010. [Last cited on 2010 Oct 15]. http://nobelprize.org/nobel_prizes/medicine/laureat es/1905/koch.html.

[6] World health Organisation (WHO). Global Tuberculosis Report. 2016 http://apps. who.int/iris/bitstream/10665/250441/1/978924156 5394-eng.pdf?ua=14.

[7] Central TB division. TB division: Revised National TB control Programme Annual Status Report. New Delhi. Directorate General of Health services, Ministry of Health and Family Welfare, Government of India, New Delhi: 2011 http://tbcindia.nic.in/ Write Read Data/TB\%20India\%202017.pdf.

[8] Swaminathan. 5GF-Opening. World Health Organization, 2016. Global Tuberculosis Control Report. Geneva: World Health Organization (WHO/HTM/TB/2016.6).

[9] World Health Organization, 2011. Towards universal access to diagnosis and treatment of multidrugresistant and extensively drug-resistant tuberculosis by 2015 . WHO progress report. Geneva: World Health Organization (WHO/HTM/TB/2011.3).
[10] Zignol M, Van Gemert W, Falzon D, et al. Surveillance of anti-tuberculosis drug resistance in the world: an updated analysis, 2007-2010. Bull World Health Organ 2012;90(2):111-9D.

[11] Matteelli A, Centis R, D'Ambrosio L, et al. Multidrugresistant tuberculosis today. Bull World Health Organ 2012;90(2):78.

[12] Raviglione M, Marais B, Floyd K, et al. Scaling up interventions to achieve global tuberculosis control: progress and new developments. Lancet 2012;379(9829):1902-13.

[13] Policy statement: automated real-time nucleic acid amplification technology for rapid and simultaneous detection of tuberculosis and rifampicin resistance: Xpert MTB/RIF system. WHO/HTM/TB/2011.4.

[14] Xpert Rapid Implementation Document: WHO Rapid Implementation of the Xpert MTB/RIF diagnostic test: technical and operational 'How-to': practical considerations. World Health Organization 2011.

[15] Alvarez-Uria G, Azcona JM, Midde M, et al. Rapid diagnosis ofpulmonary and extra pulmonary $\mathrm{TB}$ in HIV-infected patients, comparison of LED flurescent microscopy and gene expert MTB/RIF assay in a district hospital in India. Article ID 932862, Tuberc Res Treat 2012;2012: p. 4. 